\title{
Toward Robot-Assisted Dental Surgery: Path Generation and Navigation System Using Optical Tracking Approach
}

\author{
Auranuch Lorsakul and Jackrit Suthakorn* \\ Department of Biomedical Engineering, and Center for \\ Biomedical and Robotics Technology (BART LAB), Faculty of \\ Engineering, Mahidol University, Bangkok, Thailand \\ auranuch@bartlab.org and *Corresponding author: \\ egjst@mahidol.ac.th
}

\begin{abstract}
The main aim of this paper is to develop dental implant surgical navigation system based on homogenous transformation algorithms. This work is a partial section of robot-assisted surgical development. The previous works are presented in numerous basic research. They are methodology design on tool tip calibration, optical marker recognition, and pose determination using neural networks. This paper concerns with tracking path generation system based on fundamental of optical tracking. The intraoperative system is the principal focus area of this study. The homogenous transformation has been calculated in term of kinematics equation among marker relationship. The stereo camera is utilized to retrieve $3 D$ position of different pattern styles of markers. The beneath marker recognition algorithm using rotation-invariant neural network and physical method is performed to identify markers. The fundamental relationship among markers are computed to obtain the orientation and translation between the guided path and the instrument's tool tip. The experiment has been demonstrated and performed under prototype model. The method is to work on procedure step by step. They begin with patient information input and continuously perform on marker recognition, tool tip calibrations and marker digitization. The path tracking is executed to observe the accuracy of the system. The result shows that the system can be performed to track path based on beforehand planning.
\end{abstract}

Index Terms - Surgical Navigation System, Dental Implant, Optical Tracking, Computer Integrated Surgery

\section{INTRODUCTION}

Dental Implant is a surgical treatment of tooth root replacement which is the most frequently used in prosthetic dentistry. It can be used to replace missing teeth by screwing a very simple metal of medical grade such as titanium or titanium alloy into the jaw bone. A present mechanical guided system or template drill guided system is an old-fashion technology for dental implant guidance. It is derived by waxing up patient's teeth impression, and then it has to transfer the interactive planning of implants from 2D and 3D visualization to the template in reality [1]. The drawbacks of this method are on the lack of interactive control during implant is operated, as well as, the high cost of drilled guided template intervention $[2,3]$.

\author{
Chanjira Sinthanayothin and Wichit Tharanon \\ National Science and Technology \\ Development Agency (NSTDA) \\ Klong Luang, Pathumthani, Thailand \\ chanjira.sinthanayothin@nectec.or.th, and \\ wichit.tharanon@adtec.or.th
}

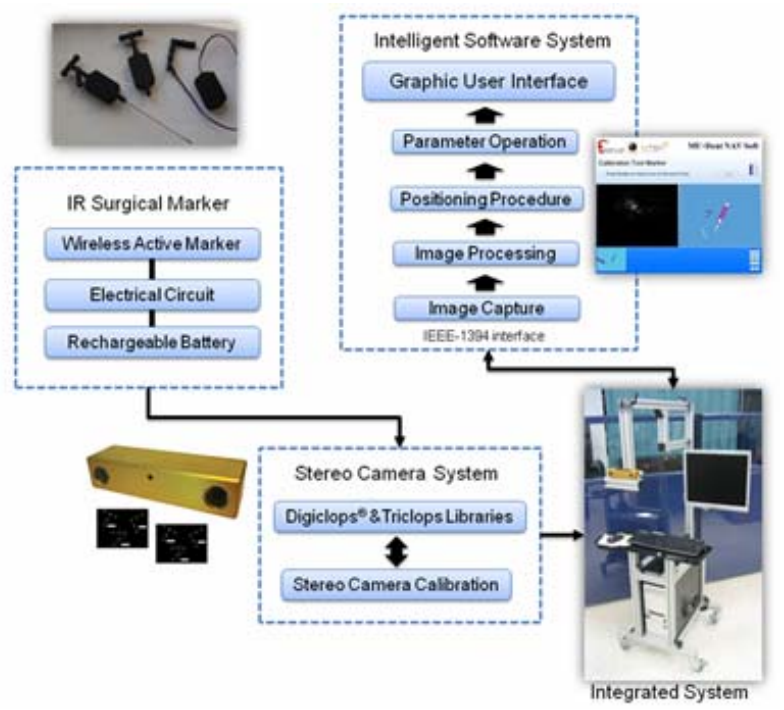

Fig. 1. Dental implant navigation system development architecture

Currently, a new system of computer-assisted surgery (CAS) for oral implantology application has been developed and introduced clinically. Computerized navigation system is offered in order to implement intraoperative guidance of the surgical instruments based on a presurgical plan. Because of its successes for the past several years, dentists have come to rely on them for solving problems that were formerly insolvable. The methodology based on CAS interventions includes preoperative and intraoperative procedures. The preoperative surgery is to use $3 \mathrm{D}$ views as provided to enhance raw images obtained from the patient before operation. The technique is to render a target region and a pathway associated with relative organs from CT data. This helps dentists to get familiar with the anatomy of the patient in advance [4]. Turning to intraoperative stage, intraoperative support can be used during the real surgical procedure in both navigation and decision aid purposes. It provides threedimensional (3D) orientation of surgical instrument position and trajectory displayed on a monitor in real-time within patient's 3D imaging data [5]. A robust and high accuracy tracking system are main parameters in navigation system. Sets of infrared (IR) based surgical marker emitters are designed in different patterns for tracking movement of surgical instruments and patient's position. Because the objects except the surgical markers in the operation room 
become invisible, it is the main benefit of the infrared based marker. IR surgical marker is introduced and developed by following of image capture and image processing procedures.

The famous commercial navigation system of dental implant currently in use is employed by Image Guided Implantology (IGI) from DENX Advanced Dental Systems, Moshav Ora, Israel [6]. Casap N et. al. [7] demonstrated that the mean spatial navigation error of IGI system achieves 0.35 $\mathrm{mm}$. It allows the transfer of the preoperative plant with a deviation of less than $1 \mathrm{~mm}$ in controlled environment. With the disadvantage of its high cost investment, the system is not widely utilized in present practical clinical treatments. Furthermore, Xiaojun C et. al. [2] cited on a computer-aided oral implantology system to expose the framework, modeling, preoperative 3D planning, registration method, optical tracking systems and real-time navigation system. They use high accuracy optical tracking system called Polaris from NDI Inc. The passive markers with IR-LED reflective element were based on photogrammetric means. In this research, it lacks experimental results and valuable discussion. Zheng G. et. al. [3] also developed computer-assisted preoperative planning and surgical navigation system in dental implantology. This system is a construction of an all-in-one system. They used NDI Polaris ${ }^{\circledR}$ Vicra $^{\mathrm{TM}}$ for real-time introsurgery guidance system. The experiment result on phantom study for this system demonstrated the mean errors of the depth, $0.772 \mathrm{~mm}$ and the angle, 0.554 degree which are acceptable for clinical trial. However, this system is necessary to perform further experiment in animal, cadaver and human. In this study, the intraoperative procedure on instrument tracking system is the main objective to study. The main focus is on optical tracking system using a stereo camera with calibration, and a set of specially designed fiducial markers attached as references on the dental hand-piece tool and patient's jaw. The task is commonly to determine the position of fiducial markers, such as infrared-emitting diodes or retroreflective spheres. They are attached on the instrument with high accuracy and minimum position tracking error. In addition, specially designed fiducial reference marker will be calculated to find the adequate length and the correct angle of the surgical tool. All retrieved important parameters are associatively registered with respect to preoperative anatomical CT data structures and surgical path planning. The benefit of this developed navigation system is to be the prototype of low cost system utilized in dental implant intervention for general patients. The main objective of paper is to introduce to algorithms concept of the integrated navigation system. The simulation demo system has been performed to confirm the concept.

\section{System Algorithm Methodology}

\section{A. System Relationship}

The proposed system is constructed by transform equation. Fig. 2 indicates a situation in which a desired transformation can be expressed as products of a chain of transformations [8]. The main objective of the intraoperative navigated system is to display the orientation and translation between the guided path and the instrument's tool tip. To calculate the position and orientation of the guided path in CT image with respect to the end-effector, $\mathbf{F}_{\text {Ctip }}$ can be computed via the kinematic equations. We can use Cartesian transforms to calculate a simple transform equation as Equation (1).

$$
\mathbf{F}_{\text {Ctip }}=\mathbf{F}_{\text {path }} \mathbf{F}_{\text {reg }} \mathbf{F}_{\text {PLM } 1}^{-1} \mathbf{F}_{C P}^{-1} \mathbf{F}_{\text {CTool }} \mathbf{F}_{\text {ToolTip }}
$$

where $\mathbf{F}_{\text {path }}$ is homogeneous transform between guided path and the stent marker reference.

$\mathbf{F}_{\text {reg }}$ is homogeneous transform between image coordinate system and the world coordinate system which can be determined by digitization method

$\mathbf{F}_{P L M 1}$ is homogeneous transform between patient marker and reference positions PLM1 (X,Y,Z)

$\mathbf{F}_{C P}$ is homogeneous transform between patient marker and the stereo camera

$\mathbf{F}_{\text {CTool }}$ is homogeneous transform between tool marker and the stereo camera

$\mathbf{F}_{\text {ToolTip }}$ is homogeneous transform between tool marker and the end-effector of the tool

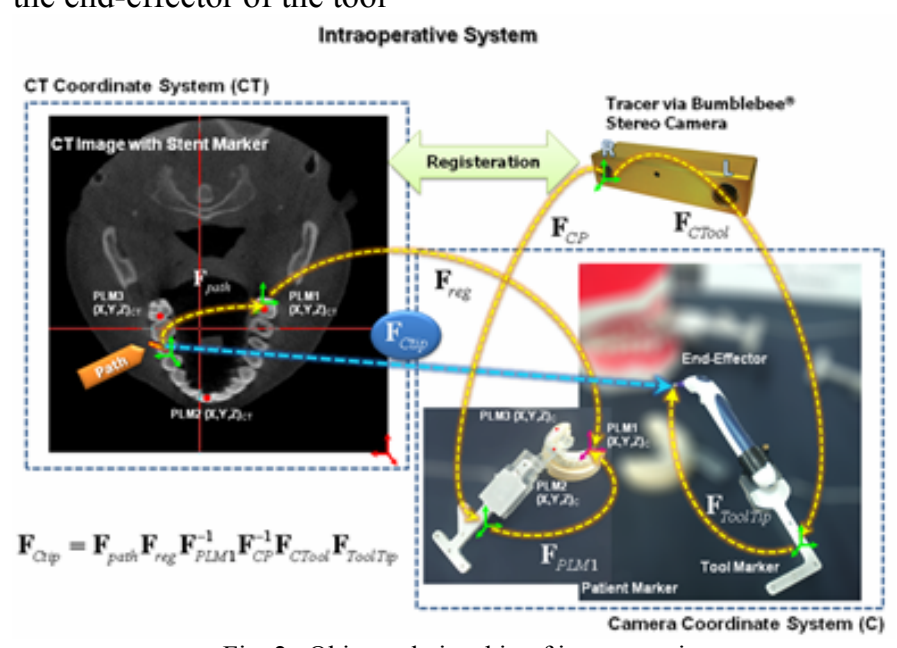

Fig. 2. Object relationship of intraoperative system.

\section{B. Sub-System Relationship}

To fulfill all over the system relationship, the position of end-effector in each device has to be determined by tool tip calibration method. This position represents the working position of the rigid body of the handpiece. It is a position that always locates the end point of the instrument wherever the movement of the tool is. The procedure of tool tip calibration will be presented in section 3.3.2 (a). Fig. 3 illustrated the relationship between each maker pattern and the stereo camera together with the tool tip relationship.

However, the relationship between the patient marker and a reference position on the patient mouth can be determined in alternative method. This relationship can be established by using a digitizer to find the linkage relationship as explained 
in Fig. 4. $\mathbf{F}_{P L M 1}$ can be calculated by the kinematic equation as following:

$$
\mathbf{F}_{P L M 1}=\mathbf{F}_{C P}^{-1} \mathbf{F}_{C D(\text { new })} \mathbf{F}_{D T i p}
$$

where $\mathbf{F}_{C P}$ is homogeneous transform between patient marker and the stereo camera

$\mathbf{F}_{C D(\text { new })}$ is homogeneous transform between digitizer marker and the stereo camera

$\mathbf{F}_{\text {DTip }}$ is homogeneous transform between digitizer marker and the end-effector of the digitizer

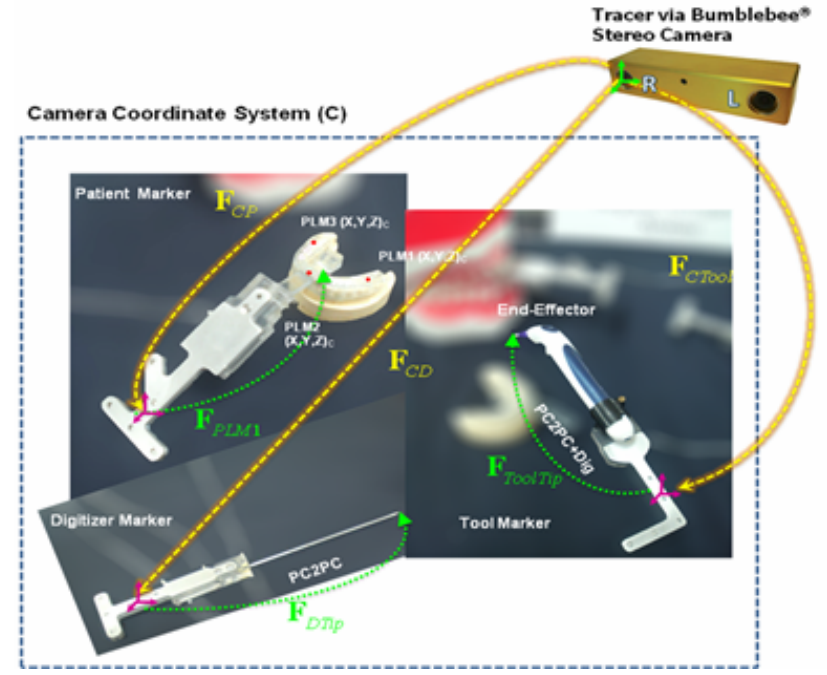

Fig. 3. Relationship between each maker pattern and the stereo camera together with the tool tip relationship

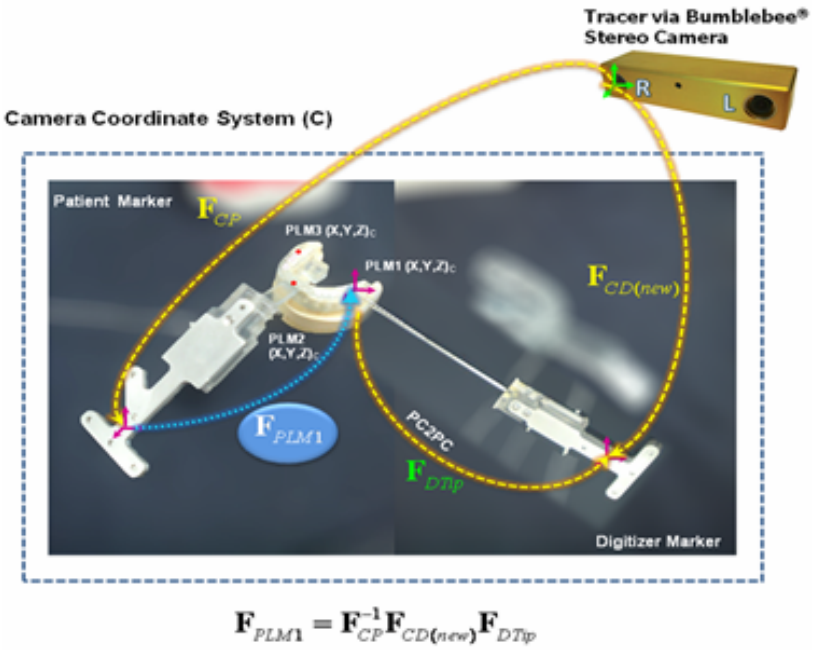

Fig. 4. Linkage relationship for the $\mathbf{F}_{P L M 1}$ homogeneous transform

\section{SyStem DEVElopMENT ARCHITECTURE}

The navigation system of the computer-assisted oral implantology system is shown in Fig. 1. The diagram is illustrated to three main major modules which are; 1) IR Surgical Marker, 2) Stereo Camera System, and 3) Intelligent Software System.

\section{A. IR Surgical Marker}

Fiducially markers used to specify positions of a hand-piece instrument, a patient's mouth and a digitizer are designed in different patterns. The active optical tracking uses Infrared light emitting diode (IR-LED) which is markers for tracked objects in the system. The markers preformed in this project consist of three patterns which are T-shape, L-shape, and Seven-shape. Prototype markers are made from aluminum that are shaped into desired patterns. At the end of each marker, there is a special jig or a clamp that helps to attach with the tools on patient's body and the instruement. IR-LEDs is utilized as a light source on the markers to form marker patterns. Each pattern differs in positions of 5 IR-LEDs, while a distance between IR-LEDs is a constant. It is set to 20 millimeters. Moreover, there is an electronics circuit for maintaining the working current to be stable even in the low voltage condition of a lithium polymer rechargeable battery. The IR markers are shown in Fig. 5.

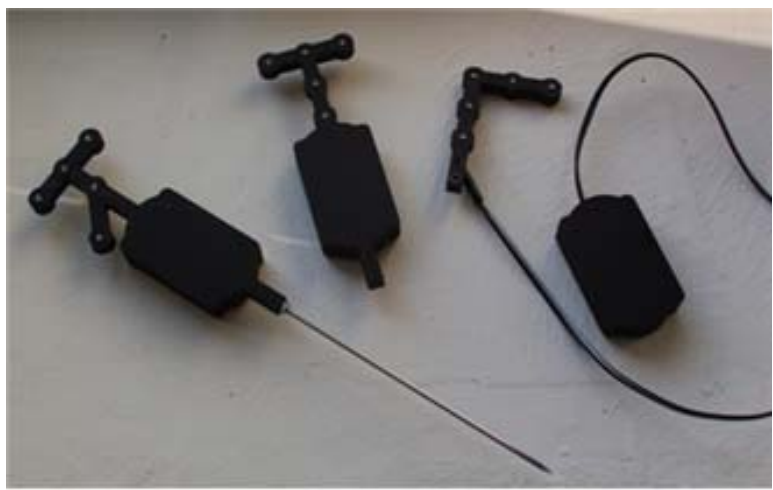

Fig. 5. IR surgical marker in three different patterns

\section{B. Stereo Camera System}

The fundamental theory of components used for 3-D coordinate acquisition system is based on pinhole camera model as shown in Fig. 6(a). The camera model interprets the image capturing process. A 3-D scene is projected onto a specific 2-D image. In addition, the principle of stereo vision tracking is currently presented in several medical navigations. The body or object to be tracked is equipped with markers that are often covered with fiducial or retroreflective surfaces. While scanning a certain volume, the tracking cameras recognize these markers. Then data of the tracking cameras are handed over to processing unit to calculate all positions by using image processing. In this system, we use stereo camera, Bumblebee $2{ }^{\circledR}$ model BB2-BW-60 (monochrome, 640x480 at $48 \mathrm{FPS}, 6.0 \mathrm{~mm}$ focal length lens, two $1 / 3$ " progressive scan CCD sensors). The image acquisition has been implemented by Digiclops ${ }^{\circledR}$ and Triclops, Bumblebee library to capture monochrome (black and white) image. Mapping a 3-D scene onto an image plane is a many-to-one transformation. Depth information can be obtained by using stereoscopic imaging 
techniques. Fig. 6(b) shows stereo imaging obtained two separate image view of an object. The distance between the centers of the two lenses is called the baseline, and the objective is to find the coordinates $(\mathrm{X}, \mathrm{Y}, \mathrm{Z})$ of the point $\mathrm{W}$ having image points $\left(\mathrm{x}_{1}, \mathrm{y}_{1}\right)$ and $\left(\mathrm{x}_{2}, \mathrm{y}_{2}\right)$.

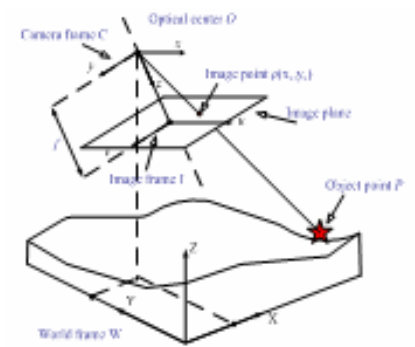

(a)

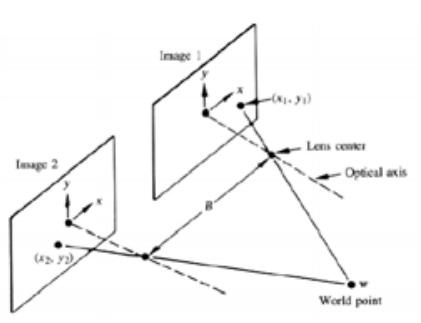

(b)
Fig. 6 (a). A pinhole camera model (redrawn [9]),

(b) Model of the stereo imaging process.

This component is the most significant module in the system. Fig. 7 shows diagram of positioning procedure. In this module, there is an image processing unit which obtains the raw image via IEEE 1394 capture card. After that the captured images are performed and calculated to achieve $3 \mathrm{D}$ coordinate positions. These positions are operated to perform marker pattern recognition, calculate frame description, and compute tool tip of each device. However, this paper will describe for 2 sections which are; 1) Image Processing and 2) Parameter Operation.

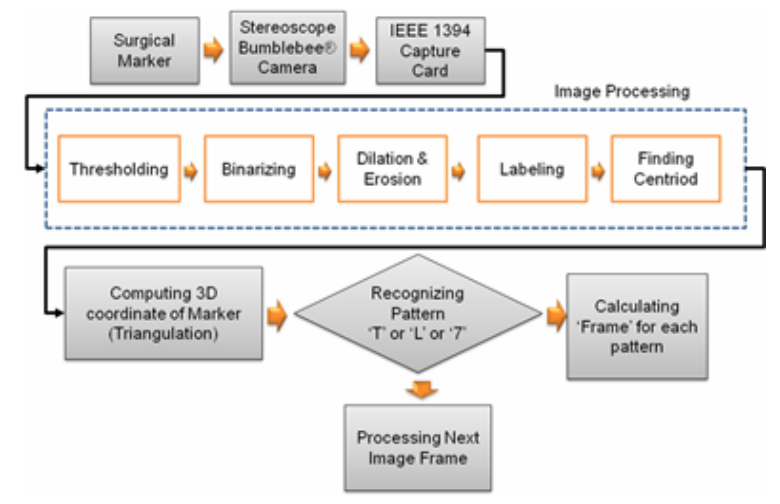

Fig. 7. Diagram of positioning procedure.

\section{1.) Image Processing:}

When the images have been acquired satisfactorily, the intended tasks are achievable with the aid in some form of image enhancement. The techniques that employed in this work are Thresholding, Dilation, Erosion, Labeling, and Finding Centroid. Each image is processed to obtain the 3D coordinates of marker positions. Thresholding and morphological image processing techniques have been performed to complete the structuring elements of object region. All centroids of marker region in left and right stereo images, and intrinsic and extrinsic stereo parameters are input into stereo triangulation function to find the $3 \mathrm{D}$ positions which are equivalent to the world coordinate system [10]. Fig. 8 shows techniques performed in image processing stage.

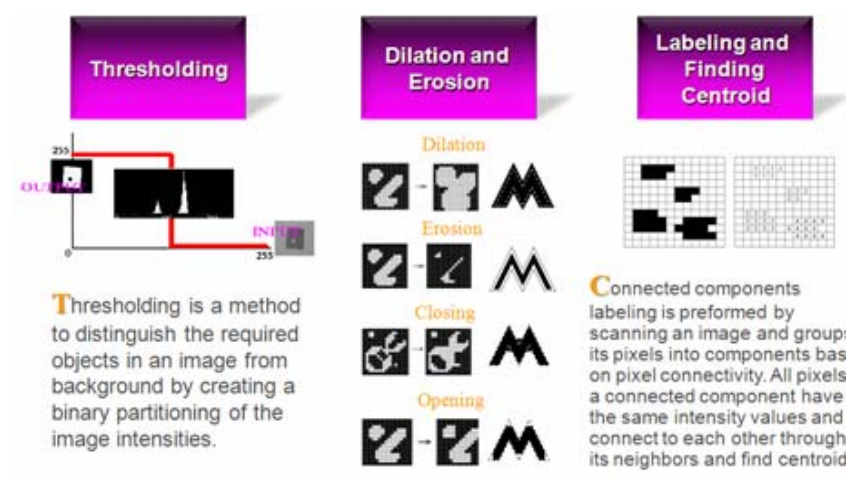

Fig. 8. Image processing stage

\section{2.) Parameter Operation}

a) Tool Tip Calibration: Dental tool tip calibrating is basically an important procedure of intraoperative surgery to determine the relationship between the hand-piece tool tip and hand-piece's markers. With the transferring coordinates from preoperative CT data to reality, this parameter is a part of components in typical registration problem. It is a part of navigation system which will be developed for further integration. A high accuracy is required, and this relationship is arranged by point-cloud-to-point-cloud rigid transformations and singular value decomposition (SVD) for minimizing rigid registration errors. For each measurement $k$, we have $\overrightarrow{\mathbf{b}}_{\text {post }}=\mathbf{R}_{k} \overrightarrow{\mathbf{b}}_{\text {tip }}+\overrightarrow{\mathbf{p}}_{k}$. With new arrangement, $\mathbf{R}_{k} \overrightarrow{\mathbf{b}}_{\text {tip }}-\overrightarrow{\mathbf{b}}_{\text {post }}=-\overrightarrow{\mathbf{p}}_{k}$.

Set up a least square problem:

$$
\left[\begin{array}{cc}
\vdots & \vdots \\
\mathbf{R}_{k} & -\mathbf{I} \\
\vdots & \vdots
\end{array}\right]\left[\begin{array}{c}
\overrightarrow{\mathbf{b}}_{t i p} \\
\overrightarrow{\mathbf{b}}_{p o s t}
\end{array}\right] \cong\left[\begin{array}{c}
\mathbf{M} \\
-\overrightarrow{\mathbf{p}}_{k} \\
\mathbf{M}
\end{array}\right]
$$

This can be represented as $\tilde{\mathbf{A}} \tilde{\mathbf{b}}=\tilde{\mathbf{p}}$ and the vector can be written as $\tilde{\mathbf{b}}=\left(\tilde{\mathbf{A}}^{T} \tilde{\mathbf{A}}\right)^{-1} \tilde{\mathbf{A}}^{T} \tilde{\mathbf{p}}$, where $\left(\tilde{\mathbf{A}}^{T} \tilde{\mathbf{A}}\right)^{-1} \tilde{\mathbf{A}}^{T}$ is pseudo inversion. This technique have been fully described in [11].

b) Optical Marker Recognition and Pose Determination Using Neural Networks: This proposed surgical marker pattern recognition and rotation angle determination for surgical navigation application. The objective is to implement a new version of tracking algorithm for marker recognition and orientation by using neural network architecture. A rotation-invariant neural pattern recognition system can recognize a rotated pattern and determine its rotation angle. The tracking system consists of markers which are indifferent patterns. The rotation-invariant neural network algorithm has to be trained by a certain quantity of 2D-Image data in various angles of rotation. Then the system is able to recognize the specific marker patterns and provide information of their 
rotation angles [12-21] shown in Fig. 9.

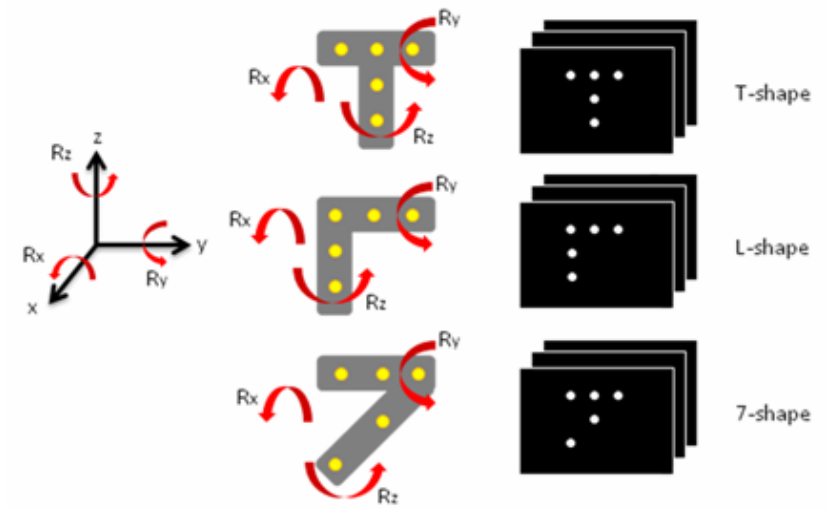

Fig. 9. Rotation angles of T-shaped, L-shaped, and Seven-shaped patterns rotate about X-Axis $\left(R_{x}\right)$, Y-Axis $\left(R_{y}\right)$, and Z-Axis $\left(R_{z}\right)$ in every $1^{\circ}$.

c) Integrated System: All parameters in an integrated navigation system are calculated in terms of depth and orientation of the dentist's tool related to patient image information. The overall diagram is presented in Fig. 10. The graphic user interface (GUI) of the system is displayed in top view, front view, lateral view and 3D view.

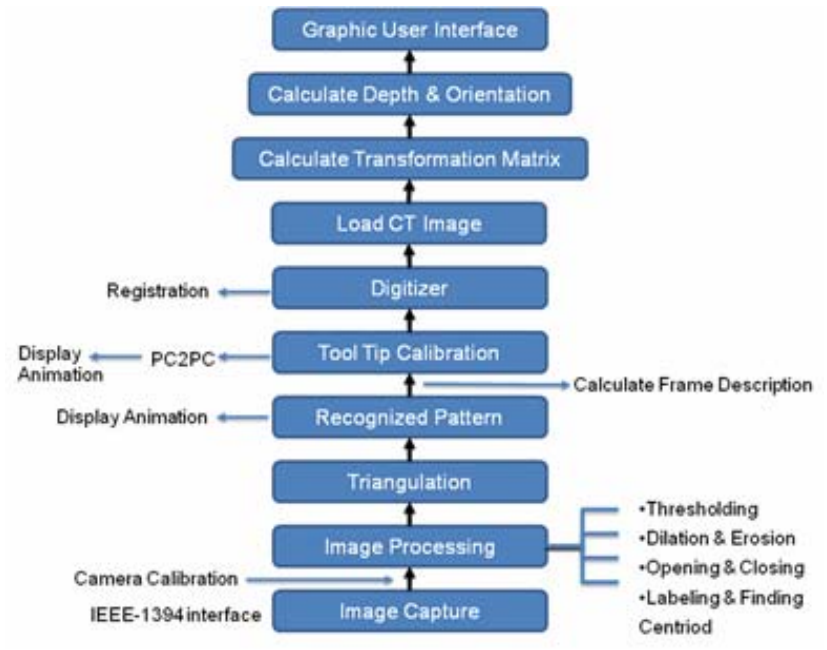

Fig. 10. Overall diagram of integrated system

\section{EXPERIMENT AND DEMO}

The integrated system is performed formerly in MATLAB $^{\circledR}$ to validate the system algorithm. The tracking system is simulated by using active marker with IR-LED in three different patterns, Bumblebee ${ }^{\circledR}$ stereo camera, and the intelligent system. The 3D coordinates, frame description, homogeneous transform, and kinemation equation are calculated respectively. Moreover, the beautiful GUI of the system is performed on C\# based system. The experiment has been performed in step by step to validate the working algorithms. The testing steps can be executed as following procedures.

Step 1: Install patient marker on the patient position

Step2: Input patient information
Step3: Recognized patient marker by program

Step4: Recognized digitizer marker by program

Step 5: Recognized hand-piece marker by program

Step6: Perform tool tip calibration for digitizer marker

Step7: Perform tool tip calibration for hand-piece marker

Step8: Use digitizer to indicate PLM1, reference position on patient stent marker

Step9: Use digitizer to indicate PLM2, reference position on patient stent marker

Step10: Use digitizer to indicate PLM3, reference position on patient stent marker

After finishing step 6 and 7, there are hand-piece and digitizer animation displayed in real time. The tool animation positions are updated related on track movement of the tools. The displayed animation is updated in real time where tool tip of the instrument is displayed in rigid body form corresponding to the marker position.

After finishing step 10, the GUI display instrument tool and digitizer animation shown as Fig. 11.

The testing is performed by controlling the instruments to track on the simulated path. The results showed that the tool of the instrument and digitizer are on the track path on the screen. This showed that the end-effector of the instruments were calibrated in good effective accuracy. Fig. 12 showed the demonstration model and instrument that used to implement on accuracy testing.

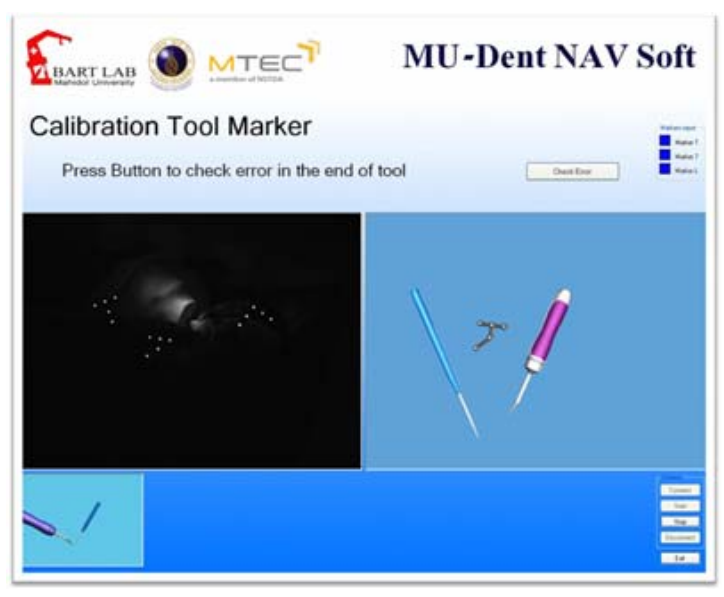

Fig. 11. GUI of the integrated system

\section{CONCLUSION}

This paper provided a systemic algorithm system about our ongoing project, the dental navigation system. The main objective of the intraoperative navigated system was to show the relationship between the guided path and the instrument's tool tip. The orientation and depth information had been calculated with respect to the end-effector of the instrument and guided path in CT images. The linkage parameters were performed by using the kinematic equations of the homogeneous transforms which were computed from the marker position in obtained images. In this work, we constructed system into 3 main parts which are 1) IR Surgical 
Marker, 2) Stereo Camera System, and 3) Intelligent Software System. Moreover, to fulfill the completed system, tool tip calibration with Point-Cloud-to-Point-Cloud Technique and the Least-Square Solution was operated to find the endeffector of tools. This work is a partial section of robotassisted surgery project which attempt to develop fully aided system on navigated, guided and assisted surgical performance. Future tasks consist of the integrated system is to do experiment with patient $\mathrm{CT}$ images both in model and the human subject to validate the accuracy of the system.

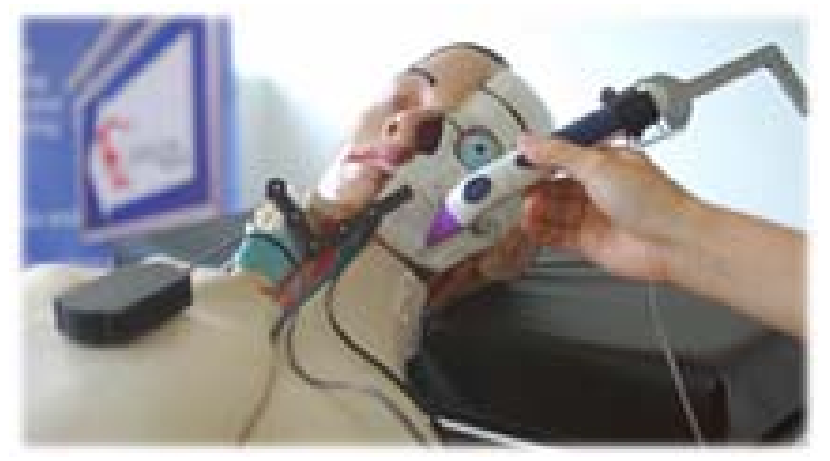

Fig. 12. Demonstration model

\section{ACKNOWLEDGMENT}

The first Author is partially supported by TGIST Scholarship (TG-11-14-49-003M), Thailand. This research is funded by MTEC, Thailand (MT-B-48-BMD-07-005-E). Special thanks to Alisa Kunapinun for interface software.

\section{REFERENCES}

[1] H. Dr.-Ing WS, "3D Planning System for Dental Implantology.."

[2] W. C. Chen Xiaojun, and Lin Yanping, "A Computer-Aided Oral Implantology System," in The 2005 IEEE Engineering in Medicine and Biology 27th Annual Conference, Shanghai, China, 2005, pp. 33123315.

[3] L. G. Guangchao Zheng, Xiaobo Li, and Jingsi Zhang, "ComputerAssisted Preoperative Planning and Surgical Navigation System in Dental Implantology," in 6th International Special Topic Conference on ITAB, 2007, Tokyo, Japan, 2007, pp. 139-142.

[4] L. Shapira, "'Image Guided Implantology" - Real-time Guidance of Dental Implant Surgery in the Operative Field using CT-scan image," CARS 2002 Computer Assisted Radiology and Surgery, pp. 959-964 June 26-29 2002.

[5] C. W. Jurgen Hoffmann, German Gomex-Roman, Siegmar Reinert, "Accuracy of navigation-guided socket drilling before implant imstallation compared to the conventional free-hand method in a synthetic edentulous lower jaw model," Clinical Oral Implantology, vol. 16, pp. 609-614, 24 November 20042005.

[6] P. N. Casap N. WA, Schnider A., and Lustmann J., "Navigation Surgery for Dental Implants: Assessment of Accuracy of the Image Guided Implantology System," American Association of Oral and Maxillofacial Surgeons, pp. 116-119, 2004.

[7] A. W. Nardy Casap, and Joshua Lustmann, "Image-Guided Navigation System for Placing Dental Iplants," Compendium, vol. 25, pp. 783-787, 2004.

[8] J. J. Craig, Introduction to Robotics Mechanics and Control: Pearson Prentice Hall, 2005.

[9] T. Kusalanukhun, "Optical Three-Dimensional Coordination for Medical Applications," Biomedical Engineering. vol. Master's Degree Salaya, Nakornpathom: Mahidol University, 2005, p. 98.
[10] F.-C. H. Chung-Hsien Kuo, and Fang-Chung Yang, "Development of Acitive IR-Based Surgical Marker Tracking and Positioning Systems."

[11] C. S. Auranuch Lorsakul, and Jackrit Suthakorn, "Point-Cloud-to-PointCloud Technique on Tool Calibration for Dental Implant Surgical Path Tracking," SPIE Medical Imaging, San Diego, CA, USA, 2008.

[12] C. S. Auranuch Lorsakul, and Jackrit Suthakorn, "Optical Marker Recognition and Pose Determination Using Neural Networks: Toward Development of A Dental Surgical Navigation System," CARS 2008 Computer Assisted Radiology and Surgery 22nd International Congress and Exhibition Barcelona, Spain, 2008.

[13] R. G. W. Bernard Widrow, and Robert A. Baxter, "Layered Neural Nets for Pattern Recognition," IEEE Transactions on Acoustics, Speech, and Signal Processing, vol. 36, pp. 1109-1118, July 1988.

[14] T. S. Chandni Patel, S. Narayan, "A Rotation-Invariant Embedded Pattern Recognition System," IEEE ICIT'02, pp. 88-92, 2002.

[15] S. Kroner, "A Neural Network for Calculating Adaptive Shift and Rotation Invariant Image Features."

[16] L. S. Max B. Reid, and Ellen Ochoa, "Rapid Training of Higher-Order Neural Networks for Invariant Pattern Recognition," pp. I-689-I-692.

[17] S. O. Minoru Fukumi, and Yosikazu Nishikawa, "Rotation-Invariant Neural Pattern Recognition System Estimating a Rotation Angle," IEEE Transactions on Neural Networks, vol. 8, pp. 568-581, May 1997.

[18] L. Fausett, Fundamentals of Neural Networks Architectures, Algorithms, and Applications. New Jersey: Prentice Hall Upper Saddle River, 1994.

[19] S. Haykin, Neural Networks A comprehensive Foundation, Second ed.: Pretice Hall International, Inc., 1999.

[20] S. Kumar, Neural Networks: A Classroom Approach: Mc Graw Hill, 2005.

[21] W. K. Ludwing Adams, Dietrich Meyer-Ebrecht, and Rainer Rueger, "Computer-Assisted Surgery," IEEE Computer Graphics \& Applications, pp. 43-51, May 1990. 